\title{
Role of Penetration Enhancers in the Topical Delivery of Adapalene by Transfersomal Gel: An in vitro Investigation
}

\author{
Akhilesh Dubey, Relma Furtado, Pratika Bhandary, Srinivas Hebbar, Amitha Shetty* \\ Department of Pharmaceutics, NGSM Institute of Pharmaceutical Sciences, Nitte (Deemed to be University), Mangaluru, INDIA.
}

\begin{abstract}
Objectives: To formulate and investigate the role of various penetration enhancers in the transfersomal gel delivery of adapalene. Methods: Transfersomes were formulated by reverse-phase evaporation method and the influence of various parameters was investigated and optimized for particle size, PDI, zeta potential, and entrapment efficiency using the Design of Experiment approach. Optimized formulation was subjected to various characterizations such as Fourier Transform Infrared Spectroscopy, turbidity measurement, and deformability study, and then transfersomes were incorporated into a suitable gel (Carbopol 934). The transfersomal gel was then characterized for gel characteristics such as $\mathrm{pH}$, spreadability, viscosity, homogeneity, extrudability, etc. Appropriate essential oils used as natural permeation enhancers like eucalyptus oil, menthol, peppermint oil, and turpentine oil were included in the transfersomal gel of adapalene, and then in vitro drug release, ex vivo permeation, and flux study was conducted and compared. Results: The adapalene loaded transfersomal gel showed particle size of $152.1 \mathrm{~nm}$, PDI of 0.315 , the zeta potential of $-42.9 \mathrm{mV}$, and entrapment efficiency of $58.65 \%$. FTIR studies showed no interactions among the drug, lipids, and polymers used. Among the
\end{abstract}

essential oils used as the permeation enhancers, the formulations containing peppermint oil displayed better in vitro drug release and ex vivo permeation than the other formulations containing remaining essential oils. Conclusion: From the present study, it can be concluded that the developed formulation can enhance the topical penetration of the drug and thereby improve therapy, which certainly adds quality to the transfersomal preparation.

Key words: Transfersomes, Adapalene, Eucalyptus oil, Turpentine oil, Menthol oil, Peppermint oil.

\section{Correspondence}

\section{Dr. Amitha Shetty,}

Assistant Professor, Department of Pharmaceutics, NGSM Institute of Pharmaceutical Sciences, Nitte (Deemed to be University), Deralakatte, Mangaluru-575018, INDIA.

Email id: amithashetty@nitte.edu.in

DOI: 10.5530/jyp.2021.13.49

\section{INTRODUCTION}

Human skin is considered the main route for topical drug delivery, and it is a readily accessible organ of the human body for topical administration. ${ }^{1}$ Skin is a permeation barrier with a low permeation rate, yet, it is one of a kind delivery pathway for therapeutic and other active agents. These compounds penetrate intercellular, intracellular, and transappendageal routes, causing transdermal delivery and topical delivery. ${ }^{2}$ Due to the effective barrier properties of the intact skin, a variety of molecules that can attain therapeutic amounts at their target site after applying to the skin is inadequate in the stratum corneum. ${ }^{3}$

Numerous formulations have been designed and utilized to overcome the permeation problem related to this route. Among the various drug delivery systems, the Nano-vesicular system has received much attention from global researchers to deliver the active moiety via the skin route. A carrier system called "Transfersomes" is introduced from the basket of the vesicular system, which has been specially designed to target skin. ${ }^{4}$ Transfersomes are completely topical formulations and are also called flexible liposomes because they can deform after applying a certain amount of stress and reform soon after releasing the stress. They are capable of delivering drugs having both low as well as high molecular weight drugs with enhanced penetration power and greater flexibility. ${ }^{5}$ The term 'Transfero' originated from the Latin word meaning to carry across and the Greek word 'soma' for a body. Transfersomes helps in overcoming the difficulty of skin penetration by compressing themselves against the intracellular sealing lipid of the stratum corneum. ${ }^{6}$ Transfersomes can spontaneously penetrate the intact stratum corneum through intracellular or trans-cellular routes and retain their shape owing to the existence of edge activators in the formulation. ${ }^{7,8}$

Retinoids used for acne therapy include tretinoin, tazarotene, adapalene, and isotretinoin (systemic). Topical retinoids will target the abnormal proliferation and diversity of keratinocytes and also will have antiinflammatory effects. Topical retinoid is comedolytic and effectively prevents the formation of micro- comedones that acts as a precursor for all the lesions of acne. First-generation retinoids (retinol, isotretinoin and, tretinoin) irritate the skin and therefore reduce patient compliance. Adapalene belongs to third-generation retinoids with minimum side effects and has been widely used because of its efficacy and less irritability to the skin compared to other topical retinoids. Topical retinoids are used as the primary treatment for most forms of acne vulgaris. The purpose of the treatment of acne is to reduce discomfort due to inflamed lesions, improve appearance, and prevent scars.

In pharmaceutical industries, natural permeation enhancers belong to the new class of penetration enhancers. Owing to their several advantages, like low cost and better safety profile, more research needs to be focused on developing stable topical formulations containing natural permeation enhancers, which can be scaled up for commercial topical drug products. ${ }^{9}$ Penetration enhancers act by one or more of the three possible mechanisms as per the lipid-protein partitioning theory. First of all, penetration enhancers change the intercellular structure of lipids in the middle of the corneocytes to enhance their diffusivity. Secondly, they can alter the

This is an open access article distributed under the terms of the Creative Commons Attribution-NonCommercial-ShareAlike 4.0 License, which allows others to remix, tweak, and build upon the work non-commercially, as long as the author is credited and the new creations are licensed under the identical terms. 
intracellular proteins inside the horny layer. Thirdly, they may increase the partitioning of the drug into the skin tissue. ${ }^{10}$

In conclusion, a penetration enhancer can increase the drug diffusivity through the skin without disrupting viable cells. The carrier should penetrate through the major skin barrier called the stratum corneum, which could also restrict the permeation of pathogens and other molecules that can enter the systemic circulation to achieve an efficient topical drug delivery. ${ }^{11}$

Therefore the study was designed to formulate an effective transfersomal topical formulation enabled with natural penetration enhancers to treat acne vulgaris.

\section{MATERIALS AND METHODS}

\section{Materials}

Adapalene was obtained from Saiyasika Biochem, Chennai. Soya phosphotidyl choline and DMSO were obtained from Himedia Lab Pvt Ltd, Mumbai. Tetrahydron Furan, Cholesterol, Sodium Deoxycholate, Ethanol, Carbopol 934, and Tween 80 are obtained from Loba Chemie Pvt Ltd, Mumbai. Chloroform and Diethyl ether is obtained from Merck India Ltd. Triethanolamine and Methanol are obtained from Nice chemicals, Edappally. Analytical grade solvents and reagents were used for the study.

\section{Methodology \\ Compatibility study by Fourier Transform Infrared Spectroscopy (FTIR) studies}

FTIR spectroscopy was performed to check the compatibility between drug, surfactants, and co-surfactants. IR spectra of soya lecithin, cholesterol, adapalene, tween 80, and sodium deoxycholate were carried out. The wavenumber of a characteristic peak of the physical mixture was compared with the pure sample and interpreted. ${ }^{12}$

\section{Formulation of transfersomal suspension}

Preparation of adapalene transfersome suspension: Transfersome was formulated by a reverse-phase evaporation method. Soya lecithin and cholesterol as a lipid phase was taken in a clean beaker and dissolved in an organic solvent such as chloroform and diethyl ether. Then tween 80 as a surfactant was poured into the previous beaker and dispersed in the mixture of organic solvent. The beaker was then placed at room temperature for a period of $24 \mathrm{~h}$ until a thin film was formed. Drug solution (adapalene $10 \mathrm{mg}$ ) was then poured over the film formed and sonicated using a probe sonicator. The film obtained was hydrated with the help of an edge activator (sodium deoxycholate) such as phosphate buffer of $\mathrm{pH} 7.4$ and was further sonicated for a minimum of $2 \mathrm{~min}$ to form a transfersomal suspension. In each suspension, dimethyl sulfoxide (DMSO) was added as a chemical enhancer. ${ }^{13}$

\section{Optimization}

The effect of process factors on the formulation of transfersomes was carried out using DOE. The concentration of soya lecithin, cholesterol, Tween 80, and sodium deoxycholate were selected as the independent variables that played an important role in the formation of vesicles. Based on the screening study, it was noted that all the other ingredients and parameters had no observable influence on the formed transfersomes and hence kept constant in further study. Optimization was performed based on the runs generated by the DOE software (Version 10.0.1; Stat-Ease). The full factorial design and the response surface method (RSM) were used in the optimization operated at 2 levels generating 30 runs. The factors selected and the levels $(-1$ and +1$)$ for the experiment are given in Table 1. The dependent variables selected were the particle size $(\mathrm{nm})$, PDI, Zeta potential $(\mathrm{mV})$, and entrapment efficiency (\%) as shown in Table 1.

\section{Characterization of transfersomes}

\section{Initial investigation of vesicle size distribution and shape}

Initial investigation on the analysis of particle size was done by BIOVIS software. After obtaining the desired results, later formulations were subjected to size and shape analysis.

\section{Drug entrapment efficiency}

The drug entrapment efficiency study was conducted to check the percentage of drug entrapped in the lipid vesicles. The study was conducted by using the cold centrifugation technique. In this method, $10 \mathrm{ml}$ of the transfersomes solution was taken into a centrifuge tube and centrifuged by cold centrifugation at $6000 \mathrm{rpm}$ at $40^{\circ} \mathrm{C}$ for a period of $2 \mathrm{~h}$. After cold centrifugation, the supernatant and sediment are separated and filtered through the $0.45 \mu \mathrm{m}$ nylon disk filter. The concentration of the adapalene $(277 \mathrm{~nm})$ in sediment and supernatant was analyzed by UV spectroscopic method, and the percentage entrapment efficiency was calculated using the following formula. ${ }^{14,15}$

\section{Turbidity measurement}

Turbidity is directly related to the concentration of surfactant or its effect in the formulation; more turbidity is more likely due to the leakage of the drug amid the vesicles. The Systronics Nephelometer determined the turbidity of the transfersomal formulation. The transfersomal formulation was transferred into $50 \mathrm{ml}$ capacity cuvettes and placed in the instrument's holder. This method has been repeated for each formulation, and turbidity measurement is displayed on the screen. ${ }^{16}$

\section{Preparation of adapalene transfersomal gel}

The transfersomal gel was formulated by formulating the 1-2 \% carbopol 934 gel. The weighed amount of carbopol was dissolved in water and kept for stirring for $2 \mathrm{~h}$. The dissolved carbopol was placed in a dark place for a period of $24 \mathrm{~h}$ for swelling. Then while stirring $10 \%$ of PEP 400 was added and kept dissolving in a magnetic stirrer, $10 \%$ isopropylene alcohol (IPA) and $10 \%$ propylene glycol were also added while stirring. Later triethanolamine was added kept undistributed to remove air bubbles. The optimized transfersome suspension was then combined with the prepared gel. ${ }^{17}$

\section{Evaluation of adapalene transfersomal gel Measurement of $\mathrm{pH}$}

The gel formulation requires maintaining the $\mathrm{pH}$ within skin $\mathrm{pH}$ for penetration; to decrease the side effects such as irritability and for absorption of the drug from a gel. The transfersomal gel $\mathrm{pH}$ was deter-

Table 1: Independent variables and dependent variables and their corresponding levels and goals used in $2^{4}$ factorial design.

\begin{tabular}{cccc}
\hline Parameters & Goal & Level $(-1)$ & Level $(+\mathbf{1})$ \\
\hline A: Soya Lecithin $(\mathrm{mg})$ & Is in range & 150 & 400 \\
B: Cholesterol $(\mathrm{mg})$ & Is in range & 25 & 100 \\
C: Tween $80(\mathrm{mg})$ & Is in range & 0 & 30 \\
D: Sodium Deoxycholate $(\mathrm{mg})$ & Is in range & 10 & 100 \\
Particle Size (nm) & Minimize & \\
PDI & Minimize & \\
Zeta Potential (mV) & Maximize & \\
Entrapment Efficiency & Maximize & \\
\hline
\end{tabular}


mined using a $\mathrm{pH}$ meter by inserting the electrode of a $\mathrm{pH}$ meter onto the surface of the gel and allowing it to equilibrate for a period of $1 \mathrm{~min}$ time. The $\mathrm{pH}$ of the optimized transfersomal gel prepared was determined in triplicate.

\section{Viscosity measurement}

Viscosity is a measurement of a fluid's resistance to flow; higher viscosity value gel is thicker, resist to flow whereas lower the value the gel is thinner and runny. The viscosity of transfersomal gel containing a percentage of water content was determined with the help of a digital Brookfield viscometer equipped with spindle No. F96 at 100 rpm speed. ${ }^{17}$

\section{Spreadability}

The special apparatus was fabricated to conduct the spreadability study of optimized gel. Spreadability is indicated in terms of time in seconds by using two slides to slip off containing the formulation in between, under the pressure of a certain load placed over it. Less time taken by the two slides to slip off, better is the spreadability. In this method, two glass of standard dimension was selected. The optimized gel, whose spreadability has to be determined is kept over one slide and the other slide is placed over the formulation across a length of $0.5 \mathrm{~cm} .100 \mathrm{~g}$ load was placed over the upper slide and is pressed uniformly to obtain a thin layer. The load was then removed and excess formulation sticking to the slide was scrapped off. The slide onto which the formulation is placed is fixed whereas the second movable slide is placed over the formulation with one end of it tied to a strand onto which load is placed using a simple pan and pulley. A $30 \mathrm{~g}$ weight was put on the pan and the time taken by the above slide to travel a distance of about $0.5 \mathrm{~cm}$ and slip off from the below slide under the pressure of the load was noted. ${ }^{18}$

The spreadability was calculated using the following formula:

$$
\text { Spreadability }=m \times l / t
$$

Where $\quad \mathrm{m}=$ load placed on the upper slide $(30 \mathrm{~g})$

$$
\begin{aligned}
& \mathrm{l}=\text { length of the glass slide }(5 \mathrm{~cm}) \\
& \mathrm{t}=\text { time taken in seconds }
\end{aligned}
$$

\section{Homogeneity}

For patient compliance, it is very essential to define the homogeneity of semi-solid formulations that are applied topically to the skin. Homogeneity was carried out by pressing a small amount of the transfersomal gel between the index finger and the thumb. The consistency determination was used to categorize the formulation as homogenous or not. ${ }^{19}$

\section{Drug content}

Drug content is done to calculate the concentration of drug present in the optimized gel, drug content of adapalene in transfersomal gel formulation was estimated. By dissolving $1 \mathrm{gm}$ of transfersome gel in the suitable solvent and vesicle was lysed by ethanol $25 \mathrm{ml}$ using sonication for about of $15 \mathrm{~min}$ and was then placed in a centrifugation tube and was centrifuged at for a period of $30 \mathrm{~min}$ at $2000 \mathrm{rpm}$. Then $1 \mathrm{ml}$ was diluted with $10 \mathrm{ml}$ of phosphate buffer $\mathrm{pH} 7.4$ and filtered. The samples were analyzed spectrophotometrically at $\lambda_{\max }$ of $277 \mathrm{~nm}$.

\section{Flux determination}

To know the deformability nature of transfersomes vesicles the flux study is conducted. The study is conducted by filtration method thus nature of elasticity of the transfersomes vesicles can be measured by flux study. The produce is forcibly extruding through the polycarbonate filter membrane with a pore diameter of 60-200 $\mathrm{nm}$ holder (stainless steel) of $25 \mathrm{~mm}$ diameter filters of $200 \mathrm{ml}$ capacity barrel at 2.5 bars. The amount or volume of vesicle suspension extruded was measured for vesicle size and shape before and also after filtering. The deformability of the vesicle membrane was determined by using the formula. ${ }^{17,20}$

$$
E=J \times\left(r_{v} / r_{p}\right)
$$

$\mathrm{E}=$ elasticity of vesicle membrane

$\mathrm{J}=$ amount of suspension which was extrude during $10 \mathrm{~min}$

$r_{v}=$ vesicles size after extrusion

$r_{p}=$ pore size of the barrier

\section{In vitro drug release study}

To find the extent and duration of release of drugs incorporated in transfersomal gel in vitro drug release study were performed using Franz diffusion cell. A control formulation and transfersomal gels with four different penetration enhancers were placed on a cellophane membrane and placed upon the vertical Franz diffusion cell. The receptor compartment contained phosphate buffer $\mathrm{pH}$ 7.4, temperature of the medium was maintained at $37 \pm 0.50^{\circ} \mathrm{C}$. Samples were collected from the receptor compartment at a pre-determined time interval. Replaced with an equal amount of buffer to maintain sink condition. With suitable dilution, absorbance was read at $277 \mathrm{~nm}$ for adapalene using a UV spectrophotometer.

\section{Ex vivo drug permeation study}

The ex vivo drug permeation of the formulation was performed using the goatskin membrane to evaluate the drug permeation. Since the structure of the goatskin membrane is similar to human skin epithelium, for ex vivo studies, the goat abdominal membrane was selected. A fresh membrane from the slaughterhouse was preserved in buffer, outer hair was removed, and the clean skin of the goat was taken and placed in the hot water for a period of $15 \mathrm{~min}$ to remove the fat used for studies. The dissolution medium used was phosphate buffer $\mathrm{pH}$ 7.4. Later the skin was placed upon the vertical Franz cell. $1 \mathrm{~g}$ of the formulated gel was taken in the membrane, which is fixed into the donor chamber. The receptor medium surface temperature was maintained at $37^{\circ} \mathrm{C}$ and stirred at $50 \mathrm{rpm}$ using a magnetic stirrer. $3 \mathrm{ml}$ were collected at the suitable interval, and absorbance was read at $276 \mathrm{~nm}$ for adapalene using a UV spectrophotometer. ${ }^{21}$

\section{RESULTS}

\section{Compatibility study}

The compatibility between pure drugs and surfactants, cholesterol was detected by FT-IR spectra (physicochemical properties of adapalene, surfactant, lipids, and gelling agents such as carbopol 937). The IR stretch between $3500-3200 \mathrm{~cm}^{-1}(\mathrm{O}-\mathrm{H})$, the stretch between 1760-1665 $\mathrm{cm}^{-1}(\mathrm{C}=\mathrm{O})$, and strong absorption bands are seen between $900650 \mathrm{~cm}^{-1}$ indicates the presence of aromatic groups. In the drug peaks, the principle peaks were approximately matched with referral principle peaks of the formulation.

\section{Formulations of transfersomal suspension}

Transfersomal suspension was formulated using a full 24 factorial design. The different ratios of lipid, surfactants, co-surfactants and their interactions using a suitable statistical tool were formulated and analyzed. Accordingly, 30 formulations were conducted and the studies like particle size, PDI, zeta potential, and percentage entrapment efficiency were conducted.

\section{Preliminary investigation of transfersomes}

Physical appearances were milky-type suspension and no phase separation was observed. 


\section{Evaluation and optimization of transfersome suspension}

The experimental design is provided in Table 2 along with the responses as observed upon experimenting as indicated by software.

\section{ANOVA and the effect of factors on the responses}

The factorial model initiated upon conducting an ANOVA analysis of the most significant factors and their interactions with each of the four responses is given in Table 1. The model equation with the lack of fit value and the perturbation plots are presented with the respective response. The optimum values of particle size, PDI, zeta potential, and entrapment efficiency are depicted in the equation and response surface plot.

\section{Effect of factors on Particle Size}

The model generated for the particle size had a $p$-value of $<0.05$ indicating the model to be significant and an $F$-value of 2.40. The difference between the adjusted ( $R 20.4032)$ and the predicted ( $R 2-0.2647)$ model $R$-squared value was less than 0.2 . The amount of soya lecithin had a major effect on the size of the particle as shown in Equation 1. The response surface is depicted in Figure 1A.

Particle size $=+397.55-68.29 \mathrm{~A}-4.33 \mathrm{~B}+40.04 \mathrm{C}-39.50 \mathrm{D}-44.99 \mathrm{AB}$ $-34.16 \mathrm{AC}+12.48 \mathrm{AD}-47.17 \mathrm{BC}-11.29 \mathrm{BD}+8.21 \mathrm{CD}-15.65 \mathrm{~A}^{2}-53.05 \mathrm{~B}^{2}$ $-46.59 C^{2}+24.49 D^{2}$

.....Equation 1

Where, A, B, C, and D represent the coded values for soya lecithin, cholesterol, Tween 80 , and sodium deoxycholate respectively.

Table 2: Experimental design and the observed responses.

\begin{tabular}{|c|c|c|c|c|c|c|c|c|c|}
\hline & & $\begin{array}{c}\text { Factor } \\
1\end{array}$ & $\begin{array}{c}\text { Factor } \\
2\end{array}$ & $\begin{array}{c}\text { Factor } \\
3\end{array}$ & $\begin{array}{c}\text { Factor } \\
4\end{array}$ & $\begin{array}{c}\text { Response } \\
1\end{array}$ & $\begin{array}{c}\text { Response } \\
2\end{array}$ & $\begin{array}{c}\text { Response } \\
3\end{array}$ & $\begin{array}{c}\text { Response } \\
4\end{array}$ \\
\hline Std & Run & A & B & $\mathrm{C}$ & D & $\begin{array}{c}\text { Particle } \\
\text { Size }\end{array}$ & PDI & Zeta Potential & $\begin{array}{c}\text { Entrapment } \\
\text { Efficiency }\end{array}$ \\
\hline & & $\mathrm{mg}$ & $\mathrm{mg}$ & $\mathrm{ml}$ & $\mathrm{mg}$ & $\mathrm{nm}$ & & $\mathrm{mV}$ & $\%$ \\
\hline 7 & 1 & 150 & 100 & 30 & 10 & 375.2 & 0.76 & -37.6 & 66.3 \\
\hline 4 & 2 & 400 & 100 & 10 & 10 & 145 & 0.364 & -42.9 & 66.92 \\
\hline 3 & 3 & 150 & 100 & 10 & 10 & 496.7 & 0.859 & -37.6 & 50.56 \\
\hline 24 & 4 & 275 & 62.5 & 20 & 145 & 368.2 & 0.884 & -49.9 & 42.3 \\
\hline 26 & 5 & 275 & 62.5 & 20 & 55 & 443.5 & 0.928 & -50.3 & 13.4 \\
\hline 12 & 6 & 400 & 100 & 10 & 100 & 166.3 & 0.551 & -36.7 & 71.7 \\
\hline 30 & 7 & 275 & 62.5 & 20 & 55 & 303.8 & 0.788 & -38 & 15 \\
\hline 25 & 8 & 275 & 62.5 & 20 & 55 & 388.4 & 0.938 & -40.5 & 18.9 \\
\hline 11 & 9 & 150 & 100 & 10 & 100 & 313.4 & 0.669 & -37.3 & 42.3 \\
\hline 6 & 10 & 400 & 25 & 30 & 10 & 262.4 & 0.394 & -43.6 & 73.5 \\
\hline 16 & 11 & 400 & 100 & 30 & 100 & 118.3 & 0.294 & -26.7 & 78 \\
\hline 21 & 12 & 275 & 62.5 & 10 & 55 & 145.6 & 0.334 & -45.4 & 38.56 \\
\hline 18 & 13 & 525 & 62.5 & 20 & 55 & 294.2 & 0.395 & -39.8 & 57 \\
\hline 8 & 14 & 400 & 100 & 30 & 10 & 128.8 & 0.364 & -40.3 & 60.28 \\
\hline 23 & 15 & 275 & 62.5 & 20 & -35 & 739.3 & 1 & -67.6 & 12.4 \\
\hline 27 & 16 & 275 & 62.5 & 20 & 55 & 325.8 & 0.627 & -43 & 49.4 \\
\hline 9 & 17 & 150 & 25 & 10 & 100 & 186.5 & 0.603 & -36.2 & 50.56 \\
\hline 29 & 18 & 275 & 62.5 & 20 & 55 & 622.1 & 0.742 & -54.4 & 48 \\
\hline 1 & 19 & 150 & 25 & 10 & 10 & 171.6 & 0.619 & -27.2 & 49.4 \\
\hline 22 & 20 & 275 & 62.5 & 40 & 55 & 393.3 & 0.835 & -51.3 & 46.7 \\
\hline 20 & 21 & 275 & 137.5 & 20 & 55 & 279 & 0.779 & -51.6 & 34.3 \\
\hline 28 & 22 & 275 & 62.5 & 20 & 55 & 301.7 & 0.761 & -48.2 & 52.6 \\
\hline 17 & 23 & 250 & 62.5 & 20 & 55 & 492.2 & 0.815 & -54.4 & 40 \\
\hline 10 & 24 & 400 & 25 & 10 & 100 & 243.6 & 0.613 & -44.8 & 67 \\
\hline 14 & 25 & 400 & 25 & 30 & 100 & 270 & 0.396 & -49.2 & 66.44 \\
\hline 2 & 26 & 400 & 25 & 10 & 10 & 265.1 & 0.627 & -48.8 & 56.68 \\
\hline 15 & 27 & 150 & 100 & 30 & 100 & 354.5 & 0.533 & -50 & 56 \\
\hline 5 & 28 & 150 & 25 & 30 & 10 & 479.1 & 0.998 & -38.9 & 43.4 \\
\hline 13 & 29 & 150 & 25 & 30 & 100 & 465.5 & 0.896 & -48.2 & 53.4 \\
\hline 19 & 30 & 275 & -12.5 & 20 & 55 & 208.2 & 0.75 & -48.4 & 61.5 \\
\hline
\end{tabular}

A- Soya lecithin, B- Cholesterol, C- Tween 80 , D- Sodium deoxycholate 

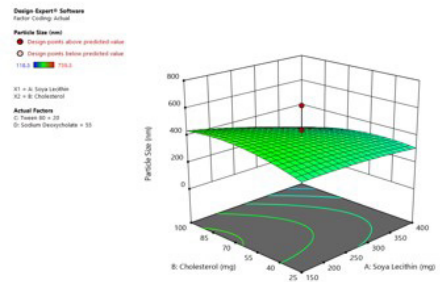

(A)
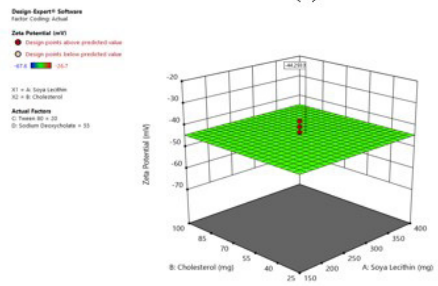

(C)
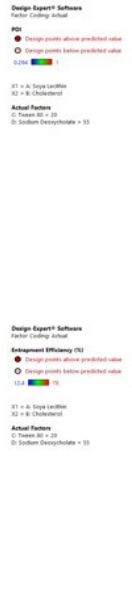

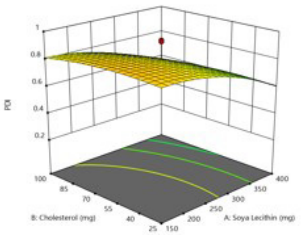

(B)

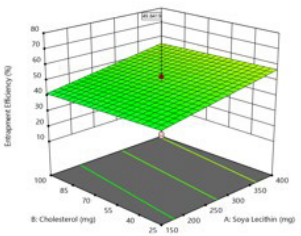

(D)
Figure 1: 3D surface response plot (A) Particle size (B) PDI (C) Zeta potential (D) Entrapment efficiency.

\section{Effect of factors on PDI}

The model generated for the PDI had a $p$-value of $<0.05$ and an F-value of 2.54. The value of 2.54 indicates a non-significant lack of fit. The difference between the adjusted ( $R 2$ 0.2671) and the predicted (R2 0.0757 ) model $R$-squared value was found to be less than 0.3 . The concentration of sodium deoxycholate had a maximum effect on the PDI as shown in Equation 2. The response surface is depicted in Figure 1B.

$\mathrm{PDI}=+0.7973-0.1322 \mathrm{~A}-0.0289 \mathrm{~B}+0.0305 \mathrm{C}-0.0276 \mathrm{D}-0.0101 \mathrm{AB}$

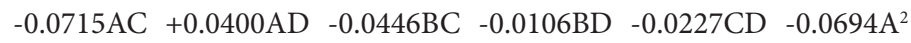
$-0.0295 \mathrm{~B}^{2}-0.0745 \mathrm{C}^{2}+0.0149 \mathrm{D}^{2} \quad$.....Equation 2 Where, A, B, C, and D represent the coded values for soya lecithin, cholesterol, Tween 80, and sodium deoxycholate respectively.

\section{Effect of factors on Zeta Potential}

The model generated for the Zeta potential had a $p$-value of $<0.05$ and an $F$ value of 1.94. The value of 1.944 indicates a non-significant lack of fit. The difference between the adjusted ( $R 2$ 0.1054) and the predicted (R2 0.4383 ) model R-squared value was less than 0.2 . The concentration of the Tween 80 did not have a major influence on the Zeta potential as shown in Equation 3. The response surface is shown in Figure 1C.

Zeta potential $(\mathrm{mV})=-44.29$

.....Equation 3

\section{Effect of factors on Entrapment Efficiency}

The model generated for the entrapment efficiency had a $p$-value of $<0.05$ and an $F$-value of 1.29. The value of 0.78 indicates a non-significant lack of fit. The concentration of soya lecithin, sodium deoxycholate, and cholesterol all had a major influence on the entrapment efficiency as shown in Equation 4. The response surface is depicted in Figure 1D.

Entrapment Efficiency $(\%)=+49.42+6.78 \mathrm{~A}-0.9467 \mathrm{~B}+2.44 \mathrm{C}-3.26 \mathrm{D}$

.....Equation 4

Where, A, B, C, and D represent the coded values for soya lecithin, cholesterol, Tween 80 , and sodium deoxycholate respectively.

\section{Optimization}

Optimization of transfersomes was for all the responses with particle size in the range of 100 to $500 \mathrm{~nm}$, PDI of 0.2 to 1 , Zeta potential of -50 to $-20 \mathrm{mV}$, and entrapment of 20 to $70 \%$. The optimized transfersomes had a particle size of $149 \mathrm{~nm}$ with a distribution (PDI) of 0.315 . The Zeta potential was observed to be $-42.9 \mathrm{mV}$ and entrapment of adapalene to an extent of $58.65 \%$.

\section{Turbidity}

The turbidity of the optimized formulation was determined using a nephelometer. This showed a turbidity of $345 \pm 0.003$.

\section{Formulation of adapalene transfersomal gel}

The optimized transfersome suspension loaded with adapalene has low viscosity hence it's a problem for application as a topical gel and there may be a chance of loss of medicament. Hence it is incorporated in a poly aqueous gelling agent namely carbopol 937 as the method written in the methodology section.

\section{Evaluation of adapalene transfersomal gel Rheological properties}

The $\mathrm{pH}$ of the gel was observed to fall in the range of 6.15-6.45 which is nearer to skin $\mathrm{pH}$. The spreadability of the formulation was observed to be in the range of $4-6.5 \mathrm{~cm}$. The viscosity of optimized formulations was observed to be in the range of between $148 \mathrm{cps}$ to $160 \mathrm{cps}$.

\section{In vitro drug release study}

The optimized transfersomal gel formulation and transfersomal gel with penetration enhancers like eucalyptus oil, menthol, peppermint oil, and turpentine oil were subjected to in vitro drug release studies using a cellophane membrane. The cumulative concentration of drug release was determined for each formulation. The results showed that transfersomal gel with peppermint oil had the highest cumulative concentration of drug release ( $86 \%$ ) up to $3-4 \mathrm{~h}$ when compared to the other transfersomal gel formulations ( $63 \%$ to $66 \%$ ). Also, it was observed that $70 \%$ of adapalene was released from the transfersomal gel after $7 \mathrm{~h}$. In vitro release studies are given in Figure 2.

\section{Ex vivo drug permeation studies}

Ex vivo drug permeation studies of the optimized gel were performed using modified ex vivo permeation apparatus using goatskin membrane. The permeation enabled with peppermint oil formulation was found to be $80 \%$ of the drug permeation in $7 \mathrm{~h}$ compared to the other oil enabled formulation. Ex vivo drug permeation study is given in Figure 3.

\section{Flux determination of transfersomal gel}

As depicted in Table 3, the steady-state flux was higher in transfersomal gel with Peppermint oil than in the optimized transfersomal gel. The

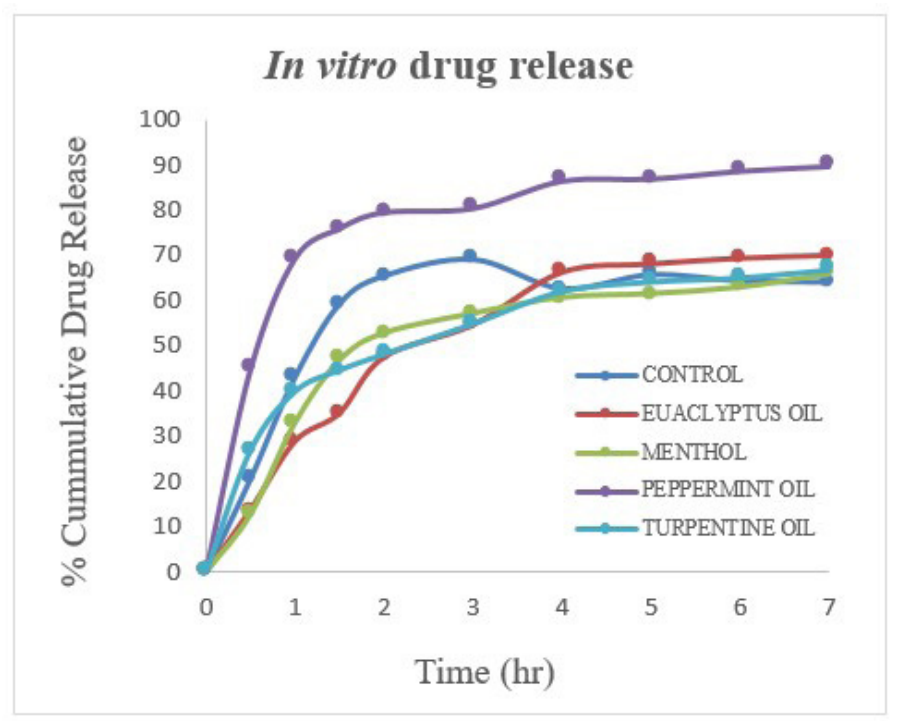

Figure 2: In vitro drug release in terms of cumulative percentage of formulation optimized formula with different penetration enhancers. 


\section{Ex vivo permeation}

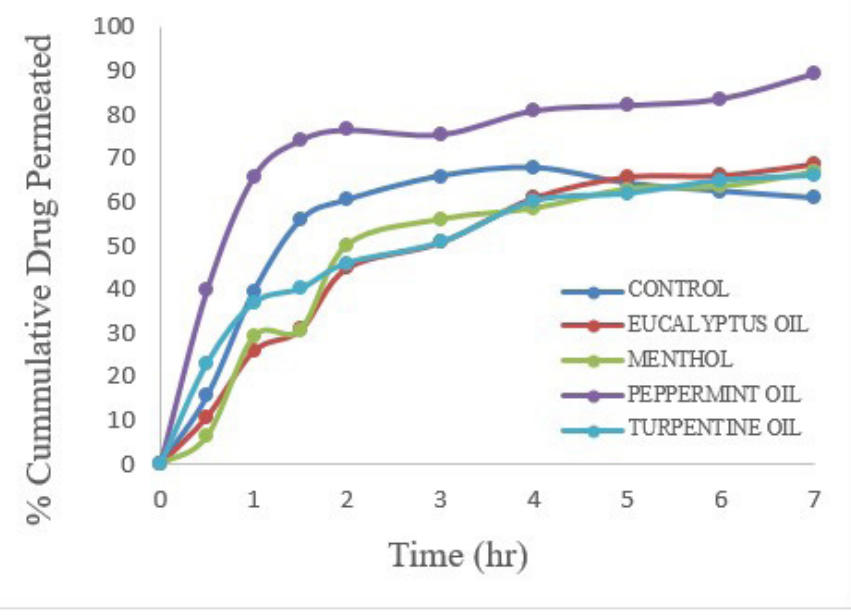

Figure 3: Ex vivo drug release in terms of cumulative percentage of formulation optimized formula.

Table 3: Permeation parameters of transfersomal gel with Peppermint oil in comparison to the optimised transfersomal gel.

\begin{tabular}{ccc}
\hline Permeation parameters & $\begin{array}{c}\text { Transfersomal gel } \\
\text { with Peppermint oil }\end{array}$ & $\begin{array}{c}\text { Optimised } \\
\text { transfersomal gel }\end{array}$ \\
\hline $\begin{array}{c}\text { Steady state flux } \\
\left.\text { (Jss } \mu \mathrm{gm}^{-2} \mathrm{~h}^{-1}\right)\end{array}$ & $85.45 \pm 0.64$ & $65.43 \pm 0.54$ \\
Permeability flux $\left(\mathrm{cm} \mathrm{h}^{-1}\right)$ & $0.0154 \pm 0.14$ & $0.0125 \pm 0.32$ \\
\hline
\end{tabular}

steady-state flux after a period of $24 \mathrm{~h}$ for transfersomal gel with peppermint oil was $85.45 \pm 0.64 \mu \mathrm{g} \mathrm{cm}^{-2} \mathrm{~h}^{-1}$, while for optimized transfersomal gel the value was $65.43 \pm 0.54 \mu \mathrm{g} \mathrm{cm}^{-2} \mathrm{~h}^{-1}$.

\section{DISCUSSION}

The findings of FTIR studies revealed the absence of any potential drug-excipient interaction and ruled out any possible physicochemical incompatibility between adapalene and excipients.

The p-value indicates the model to be significant and the $F$-value implies it to be non-significant lack of fit, indicating the model to be appropriate to calculate the size of the particle, PDI, zeta potential, and percentage entrapment efficiency. The response surface shows a reduction in the particle size when the amount of soya lecithin is increased and when the concentration of cholesterol is increased. In the case of PDI, a decline is seen as there is an increase in the concentration of sodium deoxycholate and a decrease in the concentration of Tween 80. As the amount of Tween 80 increased, no increase in the Zeta potential was seen. However, a decrease in the percentage entrapment efficiency was observed as the concentration of cholesterol and sodium deoxycholate decreased.

The turbidity value directly indicates the good number of vesicles. The outcome of the turbidity measurement studies sustained the fact that the formation of the micelles took place at higher concentrations of surfactant. Therefore, it can be finalized that turbidity of the liposomal formulation increases as the concentration of the surfactant increases. The reason for this situation may be that at low concentrations of surfactant, partition coefficient favor's the lipid phase and cause expansion of the lipid bilayer leading to increased turbidity of the vesicle dispersion. The surfactant cause fluidization of the lipid bilayer that is also responsible for the enhancement in turbidity. At optimum concentration, lipid vesicles get converted into mixed micelles, which have negligible turbidity. ${ }^{14,17}$

The $\mathrm{pH}$ evaluation is a very important parameter for topical formulations as it may irritate the skin if varied from normal skin $\mathrm{pH}$ conditions. The spreadability of the gel plays a major role in the application, if spreadability is poor then ultimately it hampers the drug residence. Viscosity is an important rheological parameter that is related to the mechanical and physical properties like spreadability, consistency, and hardness of the formulations which in turn relates to the ease of removing the product from the container, ease of application on the surface of the skin and product feel on the application site. ${ }^{5,12}$

Based on the result of drug release comparisons studies, it was seen that the rate of drug release from the optimized transfersome gel formulation was observed to be in a sustained manner. It was suggested that the spontaneous formation of a transfersome with a nano size, which permitted a controlled rate of drug release. Thus, this excellent permeability of dissolved adapalene from the transfersome formulation with peppermint oil could lead to higher absorption from the skin and be considered as an optimized formulation. In contrast, in gel without the penetration enhancers, the rate of drug release is slower and maximum within 2-4 h, which is not so effective in prolonged therapy. ${ }^{15}$

The ex vivo studies indicate peppermint oil improved the permeation by modifying the skin barrier without changing their structure, however other essential oils also improved the permeation but less than peppermint oil. ${ }^{12,14}$

A unique property associated with transfersomes is their deformability. This property, combined with the sensitivity of transfersomes to water gradient across the skin, makes transfersomes a prospective for topical application. The horny part of the skin is associated with moderately distributed pores. The pores act as permeability shunt and thereby lower the skin permeability barrier. Subsequently, these pores are also potential sites for the deformable bodies, which are strongly driven under the influence of trans-epidermal water gradient. The drug-loaded lipid suspensions are the flexibility of the vesicle. It was observed that there exists a direct relationship between the steady-state flux and the permeability coefficients. The permeability coefficient of transfersomal gel with peppermint oil also was higher than optimized transfersomal gel. The results could be accredited to higher deformability and the flexibility of transfersomes that permitted them to repress skin barrier properties. ${ }^{8,16,19}$

\section{CONCLUSION}

Transfersome of adapalene was successfully formulated by using the reverse-phase evaporation method which was then included into the gel using carbopol 934. Adapalene is third generation retinoid compound which is an analog of vitamin A having a great affinity for retinoic acid receptors (RAR) $\beta$ and RAR $\gamma$, the main mechanism of action is by inhibiting the proliferation of $P$. acnes and mainly prescribed mild to moderate acne. The major drawback with the conventional preparation is the duration of treatment and continuous application along with the side effects like dryness and pigmentation in the skin due to prolonged therapy. Hence incorporation of penetration enhancers in the transfersomes helps to achieve faster and better penetration and thereby reduces the dose frequency and duration of therapy

\section{REFERENCES}

1. Bhowmik D, Gopinath H, Pragati Kumar B, Duraivel S, Sampath Kumar KP. The pharma innovation Recent advances in novel topical drug delivery system. J Pharm Innov. 2012:1:13-31.

2. Zhang Z, Tsai PC, Ramezanli T, Michniak-Kohn BB. Polymeric nanoparticlesbased topical delivery systems for the treatment of dermatological diseases. Wiley Interdiscip Rev Nanomed Nanobiotechnol. 2013;5(3):205-18. doi: 10.1002/ wnan.1211, PMID 23386536. 
3. Chaudhary H, Kohli K, Kumar V. Nano-transfersomes as a novel carrier for transdermal delivery. Int J Pharm. 2013;454(1):367-80. doi: 10.1016/j. ijpharm.2013.07.031, PMID 23871739.

4. MVL, Zafaruddin M, Kuchana V. Design and characterization of transferosomal gel of repaglinide. Int Res J Pharm. 2015;6(1):38-42. doi: 10.7897/2230-8407.0619.

5. Al-Rohaimi $\mathrm{AH}$. Comparative anti-inflammatory potential of crystalline and amorphous Nano curcumin in topical drug delivery. J Oleo Sci. 2015;64(1):27-40. doi: 10.5650/jos.ess14175, PMID 25519291.

6. Venkatesh DN, Kalyani K, Tulasi K, Priyanka VS, Ali SKA, Kiran HC. Transfersomes: a novel technique for transdermal drug delivery. Int J Res Pharm Nano Sci. 2014;3(4):266-76.

7. Opatha SAT, Titapiwatanakun V, Chutoprapat R. Transfersomes: A Promising Nanoencapsulation Technique for Transdermal Drug Delivery. Pharmaceutics. 2020 Sep 9;12(9):855. doi: 10.3390/pharmaceutics12090855, PMID: 32916782.

8. Amnuaikit T, Limsuwan T, Khongkow P, Boonme P. Vesicular carriers containing phenylethyl resorcinol for topical delivery system; liposomes, transfersomes and invasomes. Asian J Pharm Sci. 2018;13(5):472-84. doi: 10.1016/j. ajps.2018.02.004, PMID 32104421

9. Das A, Ahmed AB. Natural permeation enhancer for transdermal drug delivery system and permeation evaluation: a review. Asian J Pharm Clin Res. 2017;10(9):5-9. doi: 10.22159/ajpcr.2017.v10i9.19389.

10. Fox LT, Gerber M, Plessis JD, Hamman JH. Transdermal drug delivery enhancement by compounds of natural origin. Molecules. 2011;16(12):10507-40. doi: 10.3390/molecules161210507.

11. Ghate VM, Lewis SA, Prabhu P, Dubey A, Patel N. Nanostructured lipid carriers for the topical delivery of tretinoin. Eur J Pharm Biopharm. 2016;108:253-61. doi: 10.1016/j.ejpb.2016.07.026, PMID 27519827.

12. Ramadon $D$, Anwar E, Harahap $Y$. In vitro penetration and bioavailability of novel transdermal quercetin-loaded ethosomal gel. pharmaceutical-sciences. 2017;79(6):948-56. doi: 10.4172/pharmaceutical-sciences.1000312.

13. Zhang YT, Xu YM, Zhang SJ, Zhao JH, Wang Z, Xu DQ, Feng NP. In vivo microdialysis for the evaluation of transfersomes as a novel transdermal delivery vehicle for cinnamic acid. Drug Dev Ind Pharm. 2014;40(3):301-7. doi:
10.3109/03639045.2012.756888, PMID 23350690

14. Jain SK, Gupta $Y$, Jain A, Rai K. Enhanced transdermal delivery of acyclovir sodium via elastic liposomes. Drug Deliv. 2008;15(3):141-7. doi: 10.1080/10717540801952407, PMID 18379926.

15. Ali MF, Salem HF, Abdelmohsen HF, Attia SK. Preparation and clinical evaluation of Nano-transferosomes for treatment of erectile dysfunction. Drug Des Dev Ther. 2015;9:2431-47. doi: 10.2147/DDDT.S81236, PMID 25995616.

16. Singh HP, Utreja P, Tiwary AK, Jain S. Elastic liposomal formulation for sustained delivery of colchicine: in vitro characterization and in vivo evaluation of antigout activity. AAPS J. 2009;11(1):54-64. doi: 10.1208/s12248-008-9078-8, PMID 19191031.

17. Vasanth S, Dubey A, G S R, Lewis SA, Ghate VM, EL-Zahaby SA, Hebbar S Development and investigation of vitamin C-enriched adapalene-loaded Transfersome gel: a collegial approach for the treatment of acne vulgaris. AAPS PharmSciTech. 2020;21(2):61. doi: 10.1208/s12249-019-1518-5, PMID 31915948.

18. Cevc G, Gebauer D, Stieber J, Schätzlein A, Blume G. Ultraflexible vesicles, Transfersomes, have an extremely low pore penetration resistance and transport therapeutic amounts of insulin across the intact mammalian skin. Biochim Biophys Acta. 1998;1368(2):201-15. doi: 10.1016/s0005-2736(97)00177-6, PMID 9459598.

19. Qushawy M, Nasr A, Abd-Alhaseeb M, Swidan S. Design, optimization and characterization of a transfersomal gel using miconazole nitrate for the treatment of candida skin infections. Pharmaceutics. 2018;10(1):26. doi: 10.3390/ pharmaceutics10010026, PMID 29473897.

20. Lu K, Xie S, Han S, Zhang J, Chang X, Chao J, Huang Q, Yuan Q, Lin H, Xu L, Shen C, Tan M, Qu S, Wang C, Song X. Preparation of a Nano emodin transfersome and study on its anti-obesity mechanism in adipose tissue of dietinduced obese rats. J Transl Med. 2014;12:72. doi: 10.1186/1479-5876-12-72, PMID 24641917.

21. Arvanitoyannis IS. Encapsulation and controlled release technologies in food systems. Int J Food Sci Technol. 2009;44(7):1462-3. doi: 10.1111/j.13652621.2008.01814.x.

Article History: Received: 14-05-2021; Revised: 07-06-2021; Accepted: 17-07-2021.

Cite this article: Dubey A, Furtado R, Bhandary P, Hebbar S, Shetty A. Role of Penetration Enhancers in the Topical Delivery of Adapalene by Transfersomal Gel: An in vitro Investigation. J Young Pharm. 2021;13(3):239-45. 